\title{
SWI/SNF-Related Matrix-Associated Actin- Dependent Regulator of Chromatin Subfamily A Member 5
}

National Cancer Institute

\section{Source}

National Cancer Institute. SWI/SNF-Related Matrix-Associated Actin-Dependent

Regulator of Chromatin Subfamily A Member 5. NCI Thesaurus. Code C19858.

SWI/SNF-related matrix-associated actin-dependent regulator of chromatin subfamily A member 5 (1052 aa, 122 kDa) is encoded by the human SMARCA5 gene. This protein is involved in both nucleosome remodeling and DNA helicase activity. 